GEOPHYSICAL RESEARCH LETTERS, VOL. 16, NO. 7, PAGES 605-608, JULY 1989

\title{
WAVEFORM COMPLEXITY IN TELESEISMIC BROADBAND SH DISPLACEMENTS: SLAB DIFFRACTIONS OR DEEP MANTLE REFLECTIONS?
}

\author{
Thorne Lay and Christopher J. Young
}

Department of Geological Sciences, The University of Michigan, Ann Arbor

\begin{abstract}
Transverse component bodywave ground displacements from deep focus earthquakes exhibit complexities which are not accounted for by standard radially symmetric Earth models. This anomalous behavior is examined using 123 broadband teleseismic SH displacement recordings, reconstituted from short- and long-period or broadband seismograms for 11 events in the Kurile subduction zone. The greatest complexity, involving an extra arrival between the $\mathrm{S}$ and $\mathrm{ScS}$ phases at epicentral distances greater than $72^{\circ}$, has a strong distance dependence and little azimuthal variation with respect to the source region, favoring an interpretation as a deep mantle triplication rather than a near-source slab diffraction.
\end{abstract}

\section{Introduction}

If the velocity structure in the lower mantle has the smooth, radially symmetric variations characteristic of a reference Earth model such as PREM [Dziewonski and Anderson, 1981], teleseismic, horizontally polarized (SH) body waves from deep focus earthquakes should be comprised only of direct waves bottoming in the lower mantle (S) or reflecting off the core $(\mathrm{ScS})$ and later arrivals involving surface reflections. Lay and Helmberger [1983] demonstrated that long-period $\mathrm{SH}$ recordings at epicentral distances greater than $72^{\circ}$ are more complicated than expected for standard Earth models. They proposed the existence of a shear velocity discontinuity about $280 \mathrm{~km}$ above the core-mantle boundary, which causes a triplication in the SH wavefield resulting in systematic waveform complexity that varies with distance from the source. Subsequently, several studies have exploited the enhanced amplitude and travel time resolution provided by broadband $\mathrm{SH}$ recordings to further examine departures from simple lower mantle velocity structure [Zhang and Lay, 1984; Beck and Lay, 1986; Choy and Cormier, 1986; Silver and Chan, 1986; Vidale and Garcia-Gonzalez, 1988].

Silver and Chan [1986] observed anomalous broadening of $S$ displacements relative to $\mathrm{ScS}$ for two deep focus events in the Kurile Islands slab at epicentral distances of $50^{\circ}$ to $70^{\circ}$. The paths to the five North American stations they used project along the strike direction of the subducting lithosphere in the Kurile Islands subduction zone. Motivated by the deep slab models for this region obtained by travel time residual sphere analysis [Creager and Jordan, 1984, 1986], Silver and Chan attributed the broadening to multipathing in a near-source slab extension into the lower mantle. Beck and Lay [1986] examined a much larger data set and found that the $S$ broadening is strongest along the slab strike and generally decreases away from this azimuth, but the broadening also fluctuates rapidly along the strike direction. Recent numerical calculations of possible slab

Copyright 1989 by the American Geophysical Union.

Paper number $89 \mathrm{GL} 00568$.

0094-8276/89/89GL-00568\$0.00 interactions indicate that diffraction rather than multipathing may explain the $S$ broadening, with the fast velocity slab acting as an anti-waveguide that distorts the $\mathrm{S}$ wavefront [Vidale, 1987; Cormier, 1989]; however, the variability in the data remains to be explained.

The investigations of broadband $S$ waveform broadening have limited their interpretations to data at distances less than $75^{\circ}$, despite clear observations of strong complexity at larger distances [Choy and Cormier, 1986; Silver and Chan, 1986]. This is because of the hypothesis by Lay and Helmberger [1983] that a lower mantle triplication causes the latter complexities; however, it is of course possible that strong slab diffractions are responsible for the anomalous arrivals beyond $75^{\circ}$, rather than deep mantle stratification. The purpose of this study is to analyze a new, high quality data set of broadband $\mathrm{SH}$ waves at distances up to $85^{\circ}$ to assess whether slab diffractions or deep mantle reflections provide a preferred interpretation of the strong waveform complexities beyond $75^{\circ}$.

\section{Data Analysis}

The data set used in this study is comprised of $123 \mathrm{SH}$ component ground displacement recordings spanning the time interval of the $\mathbf{S}$ and $\mathrm{ScS}$ arrivals. Beck and Lay [1986] previously analyzed 55 of these signals for evidence of anomalous waveform broadening. Their observations are used here to help characterize the source complexity

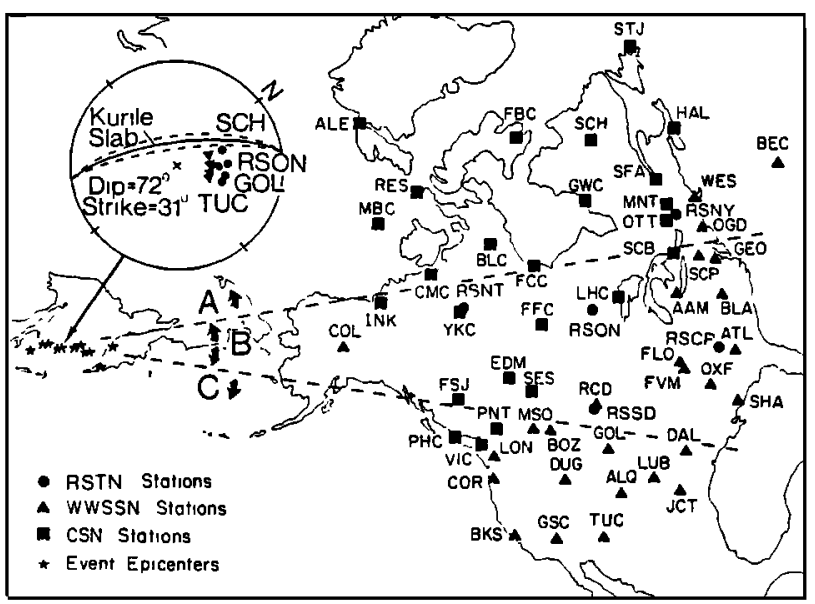

Fig. 1. Locations of deep Kurile Islands slab earthquakes and North American stations used in this study. The $20^{\circ}$ wide azimuthal sectors indicate the station groups used for the composite sections in Figure 3. The inset lower hemisphere projection shows a representative take-off angle distribution for S (solid dots) and ScS (solid triangles) phases relative to the subducting slab plane of maximum velocity anomaly from the models of Fischer et al. [1988]. The dashed lines in the lower hemisphere indicate $5^{\circ}$ variations in dip, which spans the $100 \mathrm{~km}$ thickness of the slab in the down-dip direction. 


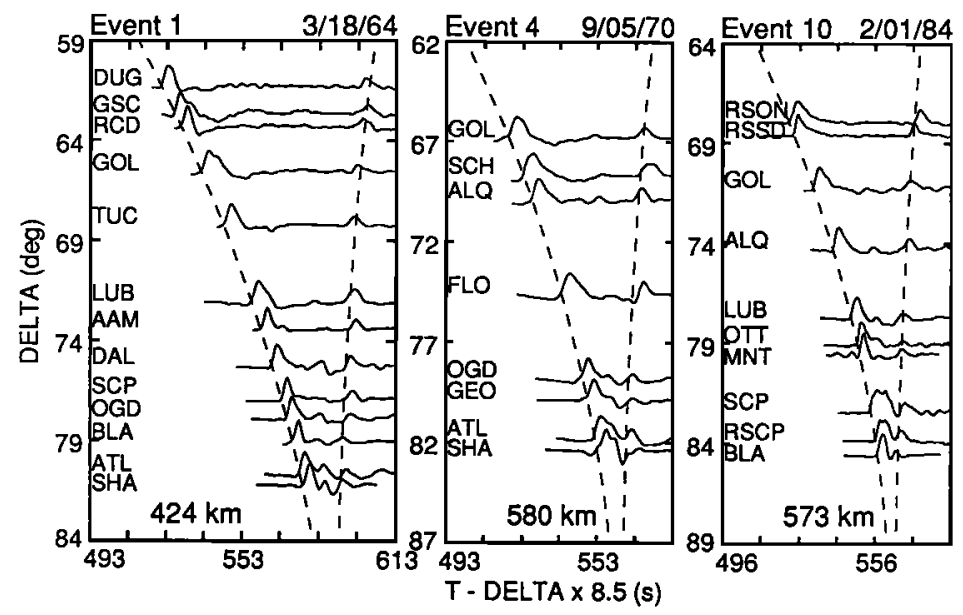

Fig. 2. Broadband $\mathrm{SH}$ ground displacement profiles for three deep events. The dashed lines indicate PREM travel time curves for S (first arrival) and ScS. The traces have been aligned on the theoretical $\mathrm{S}$ branch and amplitudes are normalized to unity.

and radiation patterns of the events. The 68 new observations are all from epicentral distances greater than $72^{\circ}$. All of the events are located in the subducting Kurile Islands slab, and the stations are the North American World Wide Standardized Seismograph Network (WWSSN), Canadian Seismic Network (CSN) and Regional Seismic Test Network (RSTN) stations shown in Figure 1. Note that the source-receiver paths straddle the strike direction of the subduction zone.

Broadband ground displacements were deconvolved from the WWSSN and CSN short- and long-period recordings using the procedure described in Beck and Lay [1986], while RSTN recordings were processed by the same method used in Silver and Chan [1986]. Not all of the stations in Figure 1 provide broadband data for each event, primarily due to variations in short-period signal quality, and a much more extensive set of all long-period recordings from 31 western Pacific earthquakes is being analyzed by Young and Lay [in preparation].

Representative profiles of SH ground displacements for three deep events are shown in Figure 2. Travel time curves for $S$ and $S c S$ for model PREM are superimposed on each profile for reference, and the first arrivals (S) have been shifted to align on the theoretical $S$ arrival branch to suppress station travel time anomalies. The ground displacement recordings from these deep focus, moderate magnitude $\left(m_{b}=5.7-6.1\right)$ earthquakes are impulsive, with two dominant pulses at each station corresponding to the $S$ and ScS arrivals. Small amplitude reverberations are apparent in the traces as well, but for the most part these are incoherent from trace to trace and constitute either ambient noise, scattered energy or reverberations at a given site.

The waveform characteristic of greatest interest for this study is the complexity which develops between the $S$ and $\mathrm{ScS}$ arrivals at distances greater than $72^{\circ}$. This involves a systematic arrival preceding $\mathrm{ScS}$ by about $15 \mathrm{sec}$ near $72^{\circ}$ and by about $5 \mathrm{sec}$ near $80^{\circ}$. This anomalous energy increases in strength with distance, resulting in maximum waveform complexity near $80^{\circ}$. Beyond $83^{\circ}$ the displacement pulses again simplify, and neither waveform broadening nor extra arrivals are seen until distances greater than $90^{\circ}$. This is the broadband equivalent of the energy modeled as a lower mantle triplication in long-period waveforms by Lay and Helmberger [1983]. The extra arrival is readily apparent in broadband, digitally recorded data such as RSCP and SCP (DWWSSN) for event 10, so the stability of the simultaneous instrument deconvolution used in processing the analog data is not in question.

Interpreting the broadband waveform complexity is not a trivial task. The data are sparse, and exhibit travel time, amplitude, source and receiver complexities which are not well understood, so any rigorous, unique inversion is precluded. We will proceed with a hypothesis testing approach. There are two classes of viable hypotheses for the waveform complexity which are not eliminated by the arguments presented by Lay and Helmberger [1983]: the first involves a deep mantle interpretation as a reflection or multipathing caused by velocity structure near the base of the mantle, and the second involves a near-source complex wave interaction with three-dimensional velocity heterogeneity extending into the lower mantle, which we collectively describe as 'slab diffraction'. Neither explanation can be precluded a priori given our present (poor) knowledge of lower mantle structure.

The most likely diagnostic between near-source and deep mantle explanations (although not a unique one) is the azimuthal and distance dependence of the anomaly. While it has been shown [e.g. Vidale, 1987] that for certain models, slab diffraction can produce a strong secondary arrival similar to the observations near $80^{\circ}$ in Figure 2, the kinematics of this arrival will be controlled by the slai orientation and velocity contrast. Using the two- and three-dimensional synthetic calculations of Vidale [1987] and Cormier [1989] as a guide, we can make the generalization that slab diffraction effects are likely to be strongest for portions of the wavefront with the longest transits along the plane of the slab. While the existence of any deep slab extension into the lower mantle below the Kurile subduction zone is controversial, the residual sphere travel time models suggest a $72^{\circ}$ dipping extension of the slab to depths of about $1300 \mathrm{~km}$ [Fischer et al., 1988]. The focal sphere inset in Figure 1 indicates the distribution of $S$ and ScS take-off angles to the North American stations used in this study relative to a hypothetical $72^{\circ}$ dipping Kurile Islands slab extension. The northernmost stations in North America should record phases with the longest transits within and along the postulated deep slab extension. 

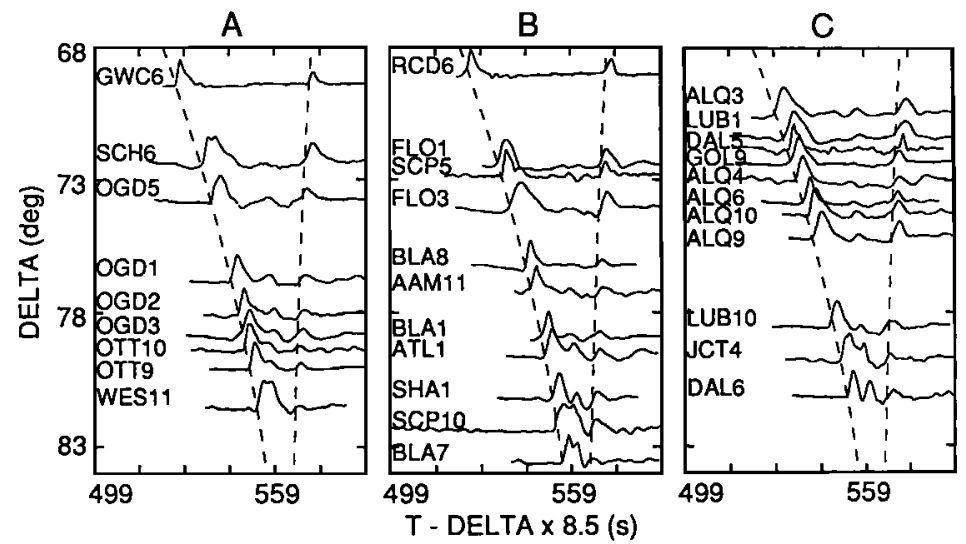

Fig. 3. Composite profiles of broadband SH ground displacements from the 11 events, in the three azimuthal sectors indicated in Figure 1. Time and distance corrections have been applied to shift the data to a reference depth of $573 \mathrm{~km}$, with corresponding S and ScS travel time curves for PREM being superimposed.

The available synthetic calculations indicate that slab diffraction effects become unimportant once the angle between the raypath and the plane of the slab exceeds about $20^{\circ}$, even for models with a deeply penetrating, strong slab anomaly like that of Creager and Jordan [1986]. For example, Cormier [1989] shows that little distortion should be observed in $\mathrm{S}$ waves at stations as little as $15^{\circ}$ away from the slab strike azimuth (away from the slab dip direction). Slab diffraction effects for more complex, distorted structures are harder to anticipate, but generally any broadening reduces the velocity gradients and thereby reduces the diffraction effects as well [Vidale, 1987]. While the three-dimensional effects are certainly not all intuitively obvious, we infer that the waveform complexity we observe beyond $72^{\circ}$ should exhibit a strong azimuthal pattern even for our restricted azimuthal coverage, if nearsource slab diffraction by a coherent slab structure like that proposed by Creager and Jordan [1986] is responsible. Alternatively, a lower mantle discontinuity should produce predominantly distance dependent complexity.

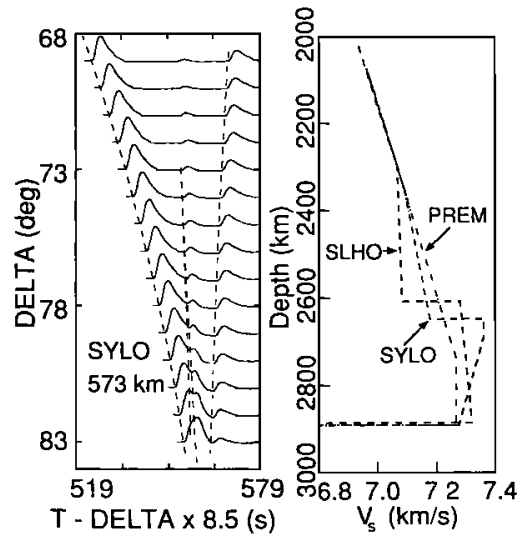

Fig. 4. Synthetic SH ground displacements for a $573 \mathrm{~km}$ deep source in a mantle with the PREM SH velocity structure above $2300 \mathrm{~km}$ depth and model SYLO velocity structure down to the core-mantle boundary. Model SYLO is shown on the right along with a PREM reference model and model SLHO of Lay and Helmberger [1983]. Travel time curves for the $S$ triplication and $\mathrm{ScS}$ arrivals for model SYLO are superimposed on the synthetics.
With this in mind, Figure 3 displays profiles of SH data grouped in the three $20^{\circ}$ wide azimuthal sectors indicated in Figure 1. The data are shifted in time and distance to correspond to a reference source depth of $573 \mathrm{~km}$, with the PREM travel time curves for $\mathrm{S}$ and $\mathrm{ScS}$ being shown. The numbers beside each station name indicate which event the observation is from. Note that each of the azimuthal sectors exhibits similar, systematic waveform complexity in the distance range $76^{\circ}$ to $82^{\circ}$. Sector A should exhibit the strongest complexity if the travel time derived slab models are correct, while sector $\mathrm{C}$ should display the least effect. $\mathrm{ScS}$ phases in sector $\mathrm{C}$ should have more complexity than $\mathrm{S}$ phases as well, given their lower projection angle relative to the hypothetical deep slab extension.

Figure 3 establishes the fact that the distance dependence of the waveform complexity is more robust than any azimuthal pattern relative to the slab, which is easier to reconcile with a deep mantle origin of the extra arrival. While reliable three-dimensional slab diffraction calculations for the full spectrum of proposed slab models have not yet been performed for paths along the slab strike to enable quantification of this argument, it is possible to make synthetic seismograms for models with deep mantle stratification to further test the latter hypothesis. Figure 4 shows a profile of synthetic SH ground displacements computed using generalized ray theory for a $573 \mathrm{~km}$ deep

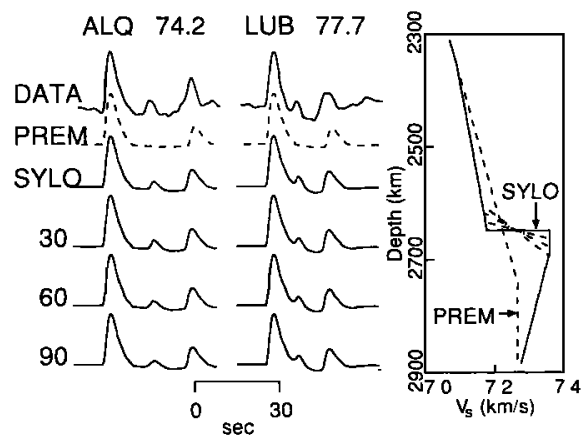

Fig. 5. Comparison between SH displacement observations at two distances for event 10 and synthetics for models PREM, SYLO, and three distributed transition zone models which are shown on the right. The numbers indicate the thickness of the transition zone for each case in kilometers. 
source in a mantle with a $2.75 \%$ shear wave discontinuity $243 \mathrm{~km}$ above the core-mantle boundary. Comparison of the distance-dependent waveform interference caused by the resulting triplication with the observations in Figure 3, indicates that such a model can explain the first-order behavior of the observations. More detailed modeling of individual events will be deferred to a future study. The discontinuity model, which is being refined using the much larger long-period data set, is similar to the SLHO model of Lay and Helmberger [1983], except for a decrease in the velocity gradient within the $243 \mathrm{~km}$ thick D" layer at the base of the mantle. The negative gradient in $D^{\prime \prime}$ is required to match long period observations at distances of $90^{\circ}$ to $95^{\circ}$. The slight decrease in gradient above the discontinuity is not resolved by the data, but does preserve accurate $S$ and $S c S$ travel times.

The broadband data allow the sharpness of the proposed discontinuity at the top of $D^{\prime \prime}$ to be constrained to a transition zone width of no more than about $30 \mathrm{~km}$, as shown by the modeling in Figure 5. This figure compares data in the triplication range to synthetics for models with distributed transition zones as well as the sharp discontinuity of model SYLO. The PREM synthetics provide references by which to isolate the triplication complexity. It is at the closer distances, near the onset of the triplication, where the data are most sensitive to the sharpness. In order to match the impulsive onset of the intermediate arrival at these distances the transition zone cannot be much thicker than 30 km.

\section{Discussion}

The limited coverage of this broadband data set, despite an order of magnitude expansion over previous data sets for distances beyond $70^{\circ}$, does not permit a unique interpretation; however, the lower mantle triplication model appears to be favored over slab diffraction effects given the lack of azimuthal variation in the data and the strong distance dependence. Is it possible to reconcile this with the travel time evidence for fast velocity slab extensions into the lower mantle? Vidale [1987] and Vidale and GarciaGonzalez [1988] have presented evidence for broadening of deep slab structure, with diminished velocity gradients giving weak slab diffractions. SH observations in Europe show little, if any, waveform complexity that would be expected for the travel time models [Cormier, 1989; Gaherty and Lay, in preparation]. Fisher et al. [1988] found that significant broadening of the slab, perhaps resulting from advective thickening during penetration into a higher viscosity lower mantle [e.g. Gurnis and Hager, 1988] can be accomodated in the travel time analysis, although the preferred slab models are for undeformed, thin slabs. The waveform effects for strongly deformed slab models need to be quantified before a final appraisal of the data in this study can be made. Of course, it should be emphasized that the travel times do not yield a unique model, and the inferred slab models may be artifacts of incomplete correction for large-scale lower mantle heterogeneity [Zhou, 1988]. Another difficulty is explaining the variability of $S$ wave broadening at closely adjacent stations [Beck and Lay, 1986] by slab diffraction effects. It may be that the notion of a coherent slab penetrating into the lower mantle as a tabular body is intrinsically oversimplified, and alternate parameterizations of the travel time inversions are needed.

The data in this study are consistent with the hypothesis of a lower mantle $S$ wave triplication, but additional analysis of a much larger data set is being conducted to determine an optimal lower mantle shear velocity model for the region of $D^{\prime \prime}$ sampled by this data. The broadband data do provide new timing and amplitude constraints which indicate that the shear velocity increase at the top of the D" layer occurs over a transition zone less than $30 \mathrm{~km}$ thick. The implications of this model are discussed in detail by Lay [1989].

Acknowledgments. This work was supported by NSF Grant EAR-8451715. Susan Schwartz helped to access the digital seismograms. We thank Gerald Schubert for organizing a stimulating special SEDI symposium at the 1988 Fall AGU meeting.

\section{References}

Beck, S. L., and T. Lay. Test of the lower mantle slab penetration hypothesis using broadband $S$ waves, Geophys. Res. Lett., 13, 1007-1010, 1986.

Cormier, V. F.. Slab diffraction of $\mathrm{S}$ waves, J. Geophys. Res., 94, 3006-3024, 1989.

Choy, G. L., and V. F. Cormier. Direct measurement of the mantle attenuation operator from broadband $P$ and $S$ waveforms, J. Geophys. Res., 91, 7326-7342, 1986.

Creager, K. C., and T. H. Jordan. Slab penetration into the lower mantle, J. Geophys. Res., 89, 3031-3049, 1984.

Creager, K. C., and T. H. Jordan. Slab penetration into the lower mantle beneath the Mariana and other island arcs of the northwest Pacific, J. Geophys. Res., 91, 35733590, 1986.

Dziewonski, A. M., and D. L. Anderson. Preliminary reference Earth model, Phys. Earth Planet. Inter., 25, 297-356, 1981.

Fischer, K. M., T. H. Jordan and K. C. Creager. Seismic constraints on the morphology of deep slabs, J. Geophys. Res., 93, 4773-4783, 1988.

Gurnis, M., and B. H. Hager. Controls on the structure of subducted slabs, Nature, 335, 317-321, 1988.

Lay, T.. Structure of the core-mantle transition zone: a chemical and thermal boundary layer, EOS, 70, 49-59, 1989.

Lay, T., and D. V. Helmberger. A lower mantle S wave triplication and the shear velocity structure of D", Geophys. J. R. Astr. Soc., 75, 799-838, 1983.

Silver, P. G., and W. W. Chan. Observations of body wave multipathing from broadband seismograms: Evidence for lower mantle slab penetration beneath the Sea of Okhotsk, J. Geophys. Res., 91, 13787-13802, 1986.

Vidale, J.. Waveform effects of a high velocity subducted slab, Geophys. Res. Lett., 14, 542-545, 1987.

Vidale, J. E., and D. Garcia-Gonzalez. Seismic observation of a high-velocity slab $1200-1600 \mathrm{~km}$ in depth, Geophys. Res. Lett., 15, 369-372, 1988.

Zhang, J., and T. Lay. Investigation of a lower mantle shear wave triplication using a broadband array, Geophys. Res. Lett., 11, 620-623, 1984.

Zhou, H.. How well can we resolve the deep seismic slab with seismic tomography? Geophys. Res. Lett., 15, 1425-1428, 1988.

T. Lay and C. J. Young, Department of Geological Sciences, The University of Michigan, Ann Arbor, MI 48109. 